AC 2007-724: REMOTE LABORATORY EXPERIMENTS: OFFERING OF A COMPLETE LABORATORY COURSE

\author{
Abul Azad, Northern Illinois University
}

Xueshu Song, Northern Illinois University 


\section{REMOTE LABORATORY EXPERIMENTS: OFFERING OF A COMPLETE LABORATORY COURSE}

\section{Introduction}

It is a challenge to provide students with an adequate laboratory experience at a time and place convenient for them. This applies to both the traditional laboratory courses as well as to laboratory courses as a part of distance learning programs. Traditional laboratory classes are scheduled only for a specified time period, when students attend a laboratory class located within their academic institution. Considering the mixed ability level of students, the allocated time is often not enough for all students to complete their tasks satisfactorily and also gain sufficient experience through the process ${ }^{1,2}$.

To address these issues, there are a number of initiatives that have been made to provide experimentation facilities over the Internet ${ }^{3,4,5,6,7,8,9}$. None of these facilities are designed to deliver a laboratory course that is a part of a regular educational program. In addition to these, all suffer from one or more of the three main drawbacks. These are: a) complexity in development, b) higher cost, and c) single server can provide access to only one experiment at a point in time. Although the Internet-based laboratory facilities have a number of potential benefits, these drawbacks hinder the process of gaining of their popularity. Towards this the authors used a unique facility to offer a regular undergraduate laboratory course within an electrical engineering technology program ${ }^{10}$. The study is conducted through a grant from the National Science Foundation (Course, Curriculum, and Laboratory Improvement program). Although, there are a number of remote facilities reported, as far as the authors' knowledge, this may be the first system of this kind that is used to offer a complete laboratory course over the Internet as a part of a regular academic program.

This paper will provide a brief description of the system, pedagogical design, laboratory implementation, and lesson learned through the laboratory offering. The next section describes remote laboratory system (in terms of hardware and software design) that was used for the remote laboratory course. Section three presents the pedagogical design of the laboratory course describing the assessment methods and implementation protocol. Section four describes the implementation details along with the system monitoring process. Section five illustrates the evaluation system that was used to assess the system itself as well as its effectiveness in terms of achieving targeted learning outcomes. These are followed by the conclusions, acknowledgement, and references.

\section{System Hardware and Software}

This section will briefly describe the overall structure of the remote laboratory facility that has been used for the study. The facility is developed in a modular structure so that each module can be changed/modified in an independent manner ${ }^{10}$. Figure 1 shows the different 
modules and their interaction. The modules are: a) Experiments; b) Interfacing; c) GUI and Web Publication; d) Server; e) Internet delivery; and f) Client PC.

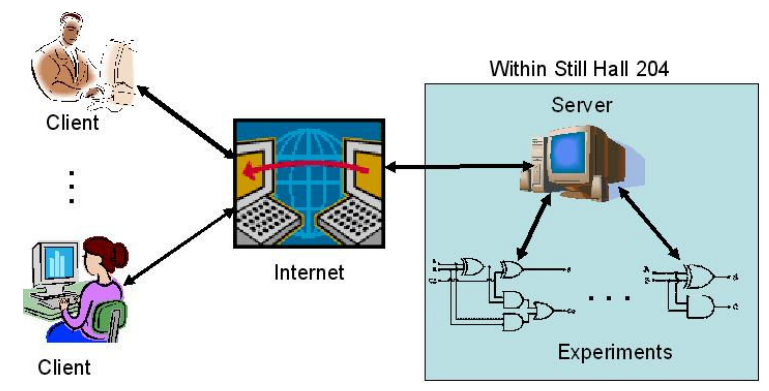

Figure 1: The block diagram of the Internet-based remote laboratory facility.

A number of experiments can be connected to the server depending upon the capability of the interfacing hardware. Each of these experiments can be controlled (manipulated) via an individual GUI residing within the server. The GUIs are developed by using National Instrument's LabVIEW software package. The GUIs are subsequently transformed into dynamic web pages and stored within the server. These GUIs (as dynamic web pages) are linked with a web application that hosts the developed facility. The clients with appropriate UserID and Password are able to access the facility and manipulate the GUIs to control an experiment. With this facility, a number of experiments can be delivered simultaneously; however, only one experiment can be accessed by a single client at any point in time. A client can access an experiment by accessing the GUI within the server. A client PC should have Internet Explorer (web browser) and LabVIEW player (freeware). In addition to performing experiments, the Internet delivery part of the facility provides documentations, user profile and password control, client access information, and weekly surveys to assess the system and its effectiveness.

Interfacing hardware and software: The first step towards the Internet-based laboratory facility is to establish an interfacing between the computer and the experiments. The computer will be the gateway to the Internet, while the experiments are the facility that needs to be accessed/operated over the Internet.

A digital Input/Output (I/O) card from National Instruments is employed as the interfacing hardware ${ }^{11,12}$. The software part of the interfacing process was implemented by using LabVIEW, which is also from National Instruments. The LabVIEW software has much more flexibility for data acquisition and control over the Internet. This can also be used along with other third party software, making it more attractive for development applications such as this one. Apart from these, the other reason for choosing LabVIEW is for its inbuilt server facility that can be utilized to publish a GUI for Internet access to the experiments ${ }^{13,14}$.

GUI and Web presentation: One of the main components of the Internet-based laboratory facility is the GUI. This is serving as the media between the experiments and the students. LabVIEW provides a facility to develop a GUI called virtual instrument (VI), which can serve both of the above purposes ${ }^{14}$. The concept of VI is to create more powerful, flexible, and cost-effective instrumentation systems using a PC. A VI can easily export and share its data and information with other software applications. 


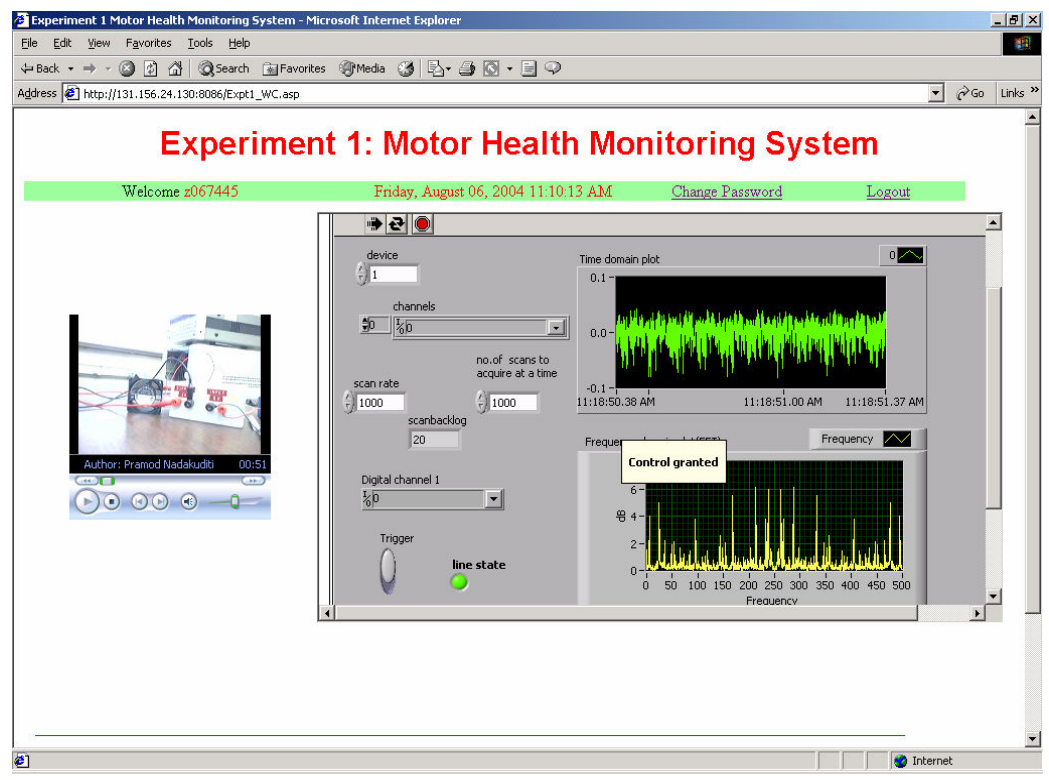

Figure 2: An image of the web page viewed by the clients from a remote location.

An image of the web page for a motor health condition monitoring experiment is shown in Figure 2. The web page consists of two individual entities. One is the GUI (with graphs and controls) and the other is the Windows Media Player panel. The GUI and the video panel are merged into this web page by using the html frames. The graphs within the GUI are presenting time and frequency domain information and can be analyzed remotely.

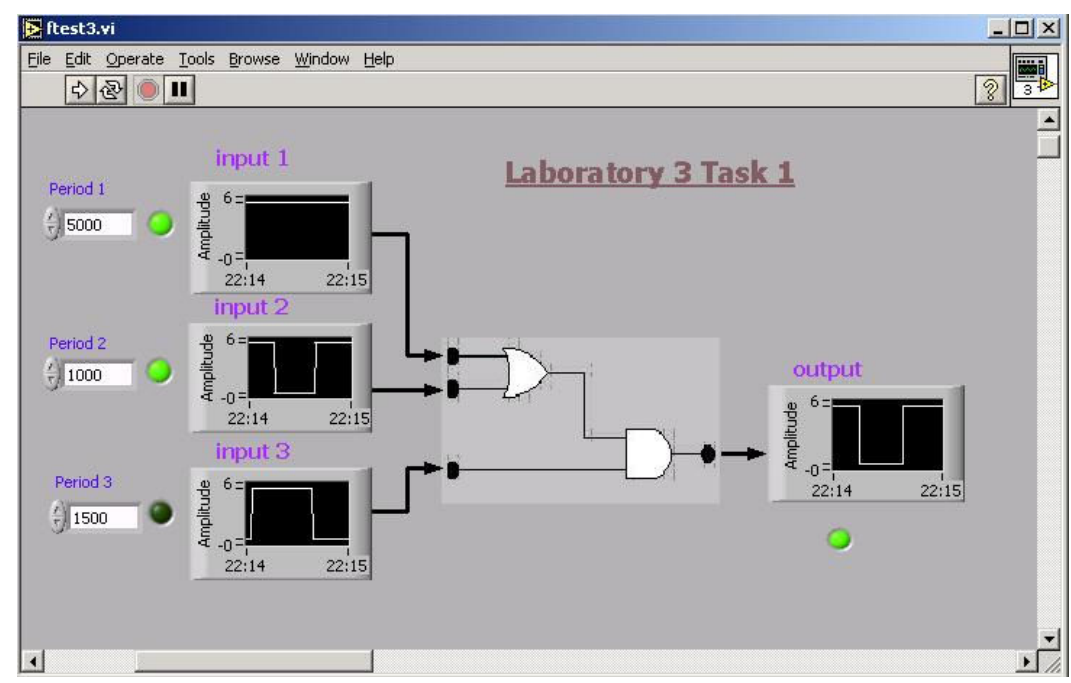

Figure 3: A GUI for a 3-input 1-output system.

An image of a GUI developed for a 3-input 1-output experiment is shown in Figure 3. The left hand side of the GUI is showing all the 3-inputs that are generated within LabVIEW.

Presenting a GUI over the Internet involves publishing the GUI as a dynamic web page. The published GUI is stored within the server at a particular location, and a web application can point the location and filename for access to the GUI. LabVIEW allows multiple numbers of 
GUIs to be published at the same time, thus allowing the system to handle multiple experiments simultaneously.

Web Server and Software Tools: A web server is hosting the web site for the facility including all the applications and interfacing hardware and software. In terms of hardware, the web server is having a $3.6 \mathrm{GHz}$ processor, $2 \mathrm{~GB}$ of RAM, $80 \mathrm{~GB}$ of $\mathrm{HD}$, and National Instrument's I/O card. For the software part, it has Windows 2003 Server (OS), LabVIEW, Internet Information Services (IIS) server, .NET, XML (EXtensible Markup Language), XSLT (EXtensible Stylesheet Language Transformations), and SQL server $2000{ }^{10}$.

LabVIEW is used for data collection and visualization. The IIS provides the services to the http requests coming through the Internet. This is a component provided within the Windows 2003 server. The IIS makes it easier to share documents and information over the Internet. Web-publishing, security, administration, and applications can work together to increase performance and reliability, while lowering the cost of ownership and also improving the web application environment. Only an authorized client with a valid password can access the system. This requires password protection and a dynamic web page. This has been implemented using ASP.NET.

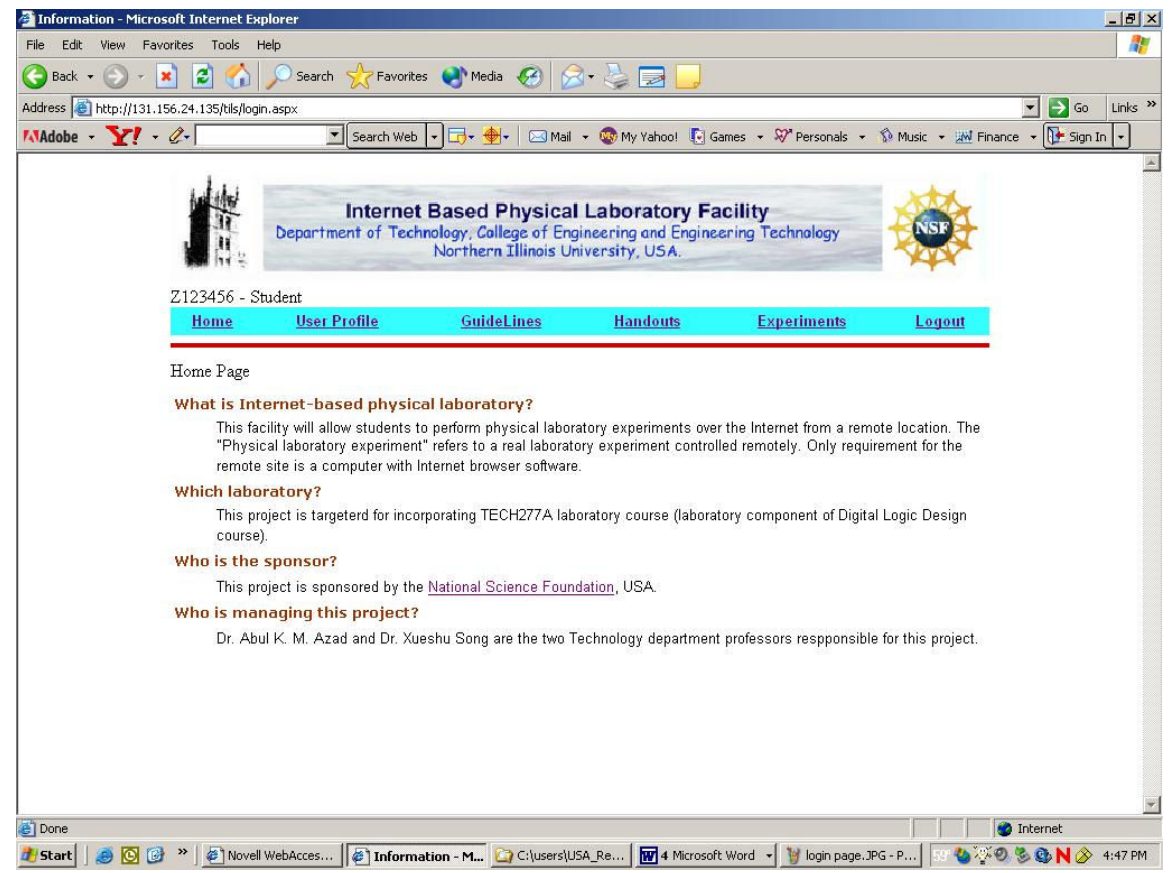

Figure 4: Homepage with client login.

Internet Delivery and Access: Internet delivery of this facility involves a number of issues: system access levels, user profile and password control, providing documentations, performing experiments, weekly surveys, and administrative activities. All these issues are addressed within the facility to make this as effective as possible. Similar to the other modules, the Internet delivery module is independent of other modules and can accept any form of experiments without any change. The only thing that has to change is the experiment related documentations. 
The system access level controls the level of access by a facility user. There will be two levels of access to the system. One will be as a client and the other as an administrator. Students will be allowed with client level access. With this status, one can perform or view an experiment, change password and demographic details, and complete the weekly survey questionnaire. An administrator level of access will allow management of experiments and monitor and gather access profile and survey data. An image of the homepage with client login is shown in Figure 4.

The UserProfile button allows the user to access demographic and contact information, the password changing facility, and computer and Internet usage information. During the first login, every user needs to answer few questions regarding their level of computer and Internet usage. This is to establish a background profile for every user. A user can change contact information and password during any login session.

There are two documentations that are provided through the web page: guidelines and handouts. The guideline document provides a brief description about the facility, its working principle, and steps to follow to perform an experiment. The handout document will provide instructions for experiments along with pre-laboratory guidelines. For the target course, the experiments and handouts are going to change every week in accordance with the delivered experiments. As a pre-laboratory, students need to study the circuit and develop a truth table before performing an experiment.

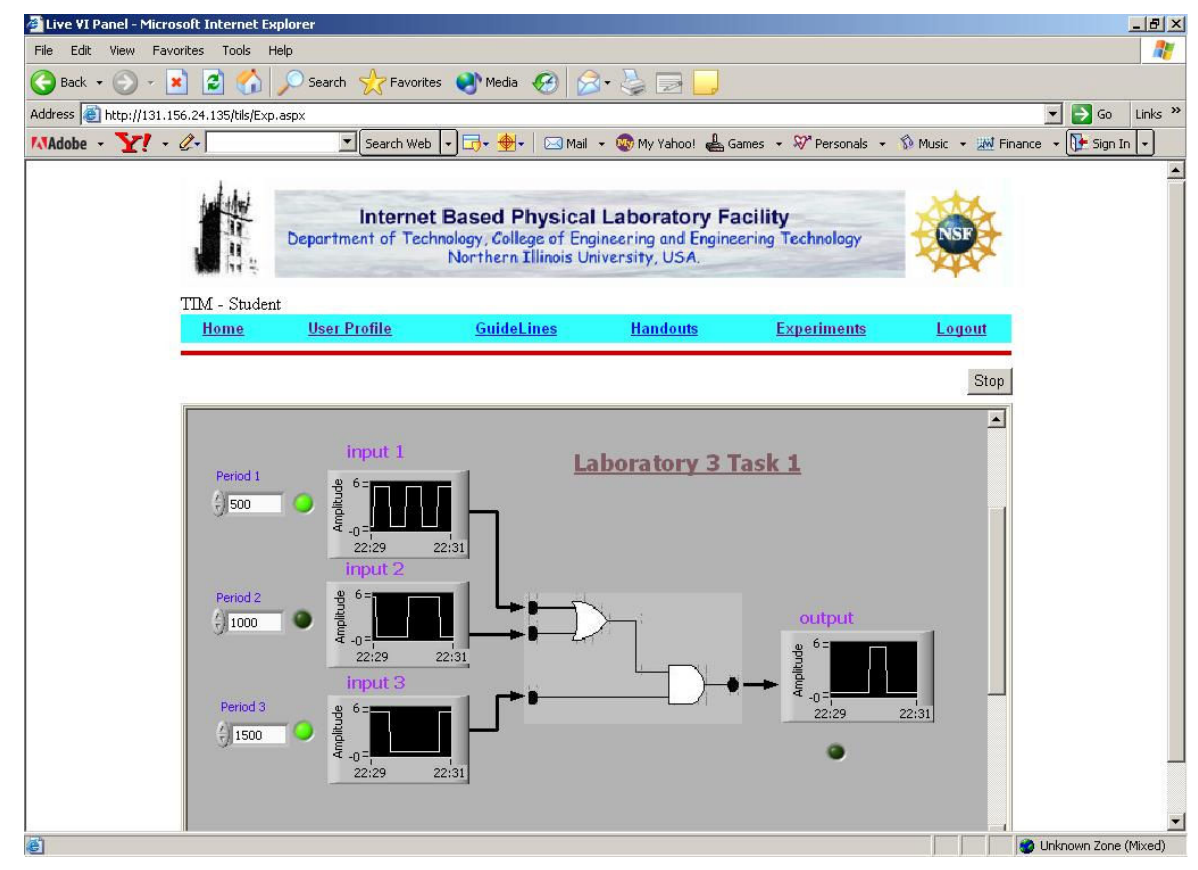

Figure 5: Image of a performer web page.

Student can access the experiments by clicking on the Experiment button within the home page. The experiment page will provide the students with a list of tasks that need to be completed during a given week. An experiment can be performed by a single user at any point in time; while other users can only view the experiment without any control over it. Depending 
upon the availability of an experiment, a client may get access either as a performer or as a viewer. An image of performer web page is shown in Figure 5. Only a performer is able to change the input status for an experiment. The viewers are put into a queue to get their turn as a performer on a first come first served basis.

\section{Pedagogical Design}

The targeted laboratory class is a complementary for a lecture class from the same discipline. The students of the targeted class will be divided into two groups (control group and test group). Half of the students are identified as the control group. The control group attended the existing traditional laboratory class settings, while the test group attended the laboratory through the remote laboratory facility. Both the control group and the test group will be developed with an even distribution of ethnic minorities and achievement levels.

For the specific course, there are a total of 12 laboratory sessions with 37 individual experiments. For each laboratory session students needs to perform certain tasks: a) completion of pre-laboratory, b) laboratory performance, and c) submission of final report. For the control group, pre-laboratory handouts were posted on the Blackboard (BB), and students need to bring the completed pre-laboratory handout when they are coming to the laboratory to attend a laboratory class. After completion of all the laboratory experiments for a laboratory session, a TA or the course teacher signs the laboratory handout. Students then prepare a post-laboratory report and submit this along with the signed pre-laboratory handout during their following week's laboratory. A format of the post-laboratory report is provided Appendix-A.

The test group also performs the same tasks but in a different way. All their activities (pre-laboratory, laboratory performance, and post-laboratory) were done through the Internet. In this case, some of the features of the available BB system are also used. The test group students were enrolled within a separate BB course. A block diagram presenting the weekly cycle of actions is shown below in Figure 6. The start button shows the starting point for the process.

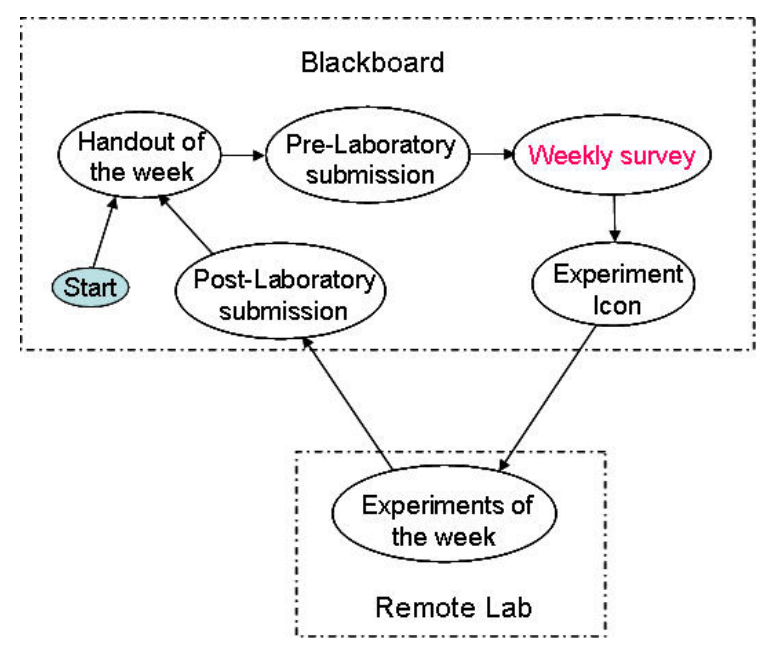

Figure 6: Remote laboratory protocol for the Internet laboratory implementation. 
The steps are listed below:

a) Handout: Handout of the week was posted on the BB. This was in MS-Word format. Students need to download this on their PC. Handouts will be posted on Thursday.

b) Pre-laboratory submission: Students need to perform the prelaboratory tasks within the handout, and after completing the pre-laboratory tasks the handout should be submitted through course drop box by Sunday.

c) Weekly survey: One of the important tasks related to the experiments is the weekly survey ${ }^{15}$. There will be no weekly survey for the first laboratory. For subsequent weeks, students need to complete a survey considering their last week's experience about the whole remote laboratory system. This survey will allow the facilitator to update the system for better performance. One can't have access to the Experiment Icon and can't perform future experiments unless completing the weekly survey.

d) Experiment of the Week: By clicking on the Experiment Icon within the $\mathrm{BB}$, student can be able to access the remote laboratory web site. One needs to perform all the tasks that are posted for each week. All the experiments need to be done by Saturday before the submission of the post laboratory report.

e) Post-laboratory submission: Post-laboratory report and updated laboratory handout were to be submitted by Sunday.

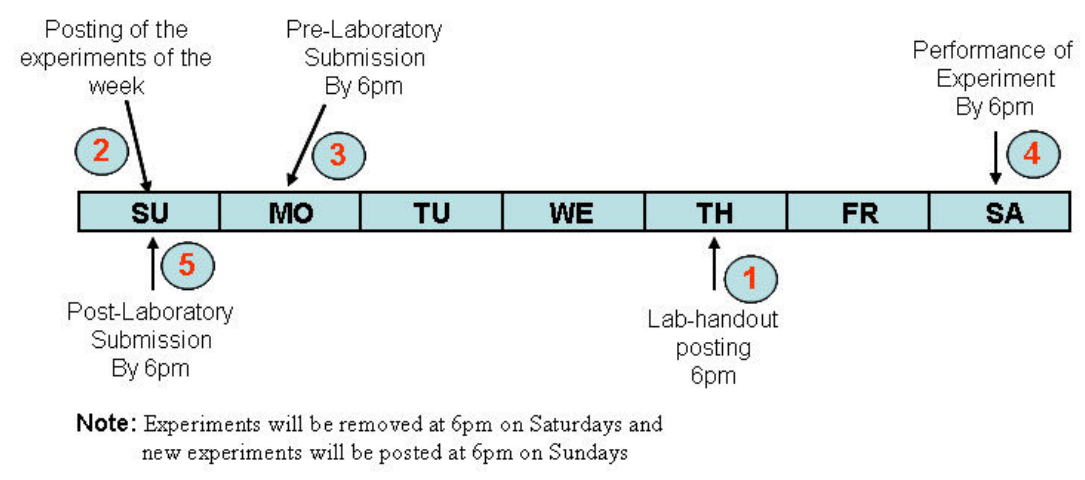

Figure 7: Weekly timeline for the remote laboratory course.

The timeline for the remote laboratory related activities is shown in Figure 7. Tasks 3, 4, and 5 were the students' responsibility, while tasks 1 and 2 are the course faculty's responsibility. The test group students are provided with a fifteen page instruction document describing the working principle of the remote laboratory, how to get access and perform experiments using the system, and all the weekly steps. 


\section{Remote Laboratory Implementation}

This section will discuss the measures that were taken for successful implementation of the remote laboratory for the target course. As mentioned earlier, this is a novel initiative towards offering a regular laboratory course as a part of an engineering technology program. The implementation design considered two important issues: a) this is a course within an undergraduate program and $b$ ) there will be very little interaction between the students and the course faculty. The measures taken to tackle these issues are:

a) A few minutes of brief weekly meeting with the test group students at the end their lecture class. This allows the students to bring up any remote laboratory related issues that have not been addressed.

b) Regular monitoring of pre- and post-laboratory submission times on the BB facility and let the respective student know if there is any late and nonsubmission.

c) Regular monitoring of experiment performance data. This allows the course faculty to ensure that each student is performing the laboratory in a timely manner.

d) An arrangement was made to ensure the performance of the weekly survey within the BB. With this, a student can view the experiment link only when he/she performed the survey. The experiment link allows one to get access into the remote laboratory facility.

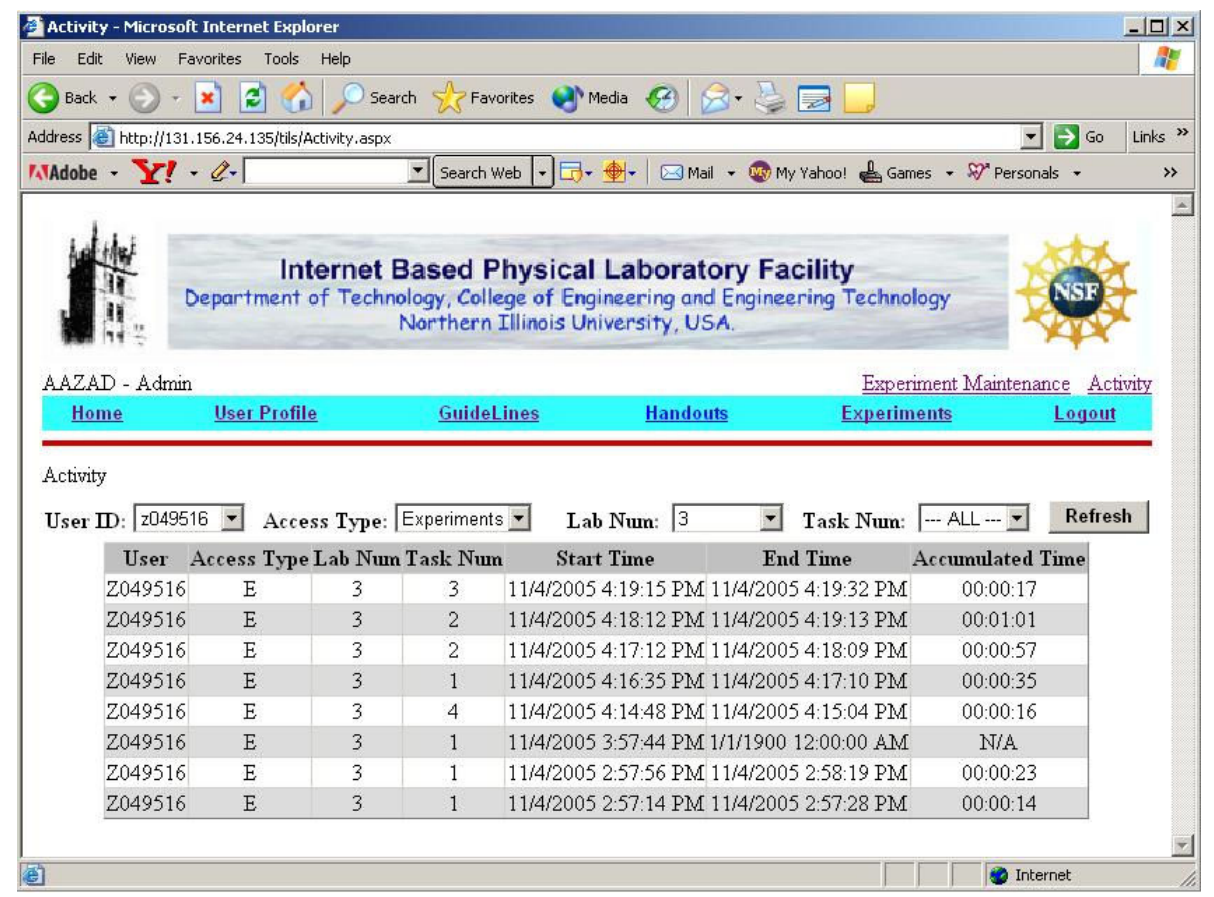

Figure 8: The activity page allows the course faculty to monitor the login and logout times.

These measures allow the authors to identify troubled students and issues and take appropriate measure to resolve the difficulties. 
The administrative level of access to the facility allows a user to have additional capabilities, such as maintenance of available experiments and gathering user activity data, and the results of weekly surveys. These application features allow an administrative user to activate or deactivate a given laboratory session or a specific task within a session at the Internet level. Activation of any experiment should be followed by the loading of appropriate GUI and connecting the hardware experiment with the facility. All these need to be synchronized to make a specific experiment available through this facility.

Considering this is $24 / 7$ facility, the system can be accessed any time from anywhere. To understand the user access profile, the system is provided with a provision to gather user activity data in terms of client login time, logout time, and performance duration for each client for a given experiment task. These data can be accessed by an administrative user through an application. An image of the activity page is shown in Figure 8. Similar to the activity data, the weekly survey data can also be gathered by an administrative user and exported to Excel for analysis. These data will allow the course administrator to use this information (in addition to other course data) towards assessment and also to study the students' learning behavior using this facility, enabling the administrator to assess the usefulness of the developed facility and adjustments/changes to make the system more efficient and effective.

\section{Evaluation}

The evaluation system has a threefold objective: a) one was to monitor the performance of the test group in relation to the control group in order to evaluate the effectiveness of the system, b) to assess the acceptability of the remote laboratory facility to the students, and c) to assess the student activity profile to understand the student habits in terms of use of the facility.

Monitoring students' performance: This is to determine the effectiveness of the project relative to the existing traditional laboratory teaching method. A number of measures were taken to evaluate the performance of both the control group and test group. These includes: to assess the post-laboratory reports and conduct of pre- and post-laboratory tests. Through the postlaboratory reports students were tested to determine their level of mastery of the subject area and their interest level in the topics and application covered. The final grade from this course will also be used as an input towards the summative evaluation process. Both groups were given with pre- and post-tests at different stages of the course and differences between the pre- and posttests are to be compared between the control group and the test group with both descriptive and inferential statistics. Similar exercises were to be performed for the obtained grade. The students' perceptions of the lasting impact of this project and the effectiveness of vertical integration will be addressed through the performance evaluation of the students during their senior years.

Acceptability of the remote laboratory facility: Towards this students are provided with a weekly survey (multiple choice), where they queried regarding their interest level in the material, adequacy of background preparation, usefulness of the handouts, effectiveness of the tutorials, the knowledge they acquired from each topic, relevance of course materials, ease of access to the Internet facility, and suggestions they have for improvement. All of this information was to be used towards quantitative analysis as well as ongoing improvement/updating of the teaching materials, experimental facility, and delivery approach. At the end of the semester, students 
were also asked to provide a qualitative assessment of the overall system and their experience from this remote laboratory facility.

Student activity profile: As an integral part of the remote laboratory system, students' activity profiles were monitored. This provision records when a student logs in or logs out to the system and also how long he/she performed an experiment. This allows the course teacher to monitor when and for how long a student access to experiment. At the same time the data can be used to develop an idea of the student habit (choice of time in performing the laboratory over the Internet).

External Evaluator: To overview the whole evaluation process Dr. Herbert J. Walberg, Ph.D. (Research Professor of Education and Psychology at the University of Illinois at Chicago and Visiting Professor at Stanford University) is acting as the external evaluator. He is a world renowned scholar, researcher in teaching psychology, and evaluation. Dr. Walberg has been advising the authors on questionnaire designs, evaluation of the pedagogical effects of the system, data analysis, and interpretation. The independent evaluator has approved the evaluation criteria, questionnaire, and method through interactions with the authors.

The laboratory course was offered during the Fall 2006 and all the data were collected and now under analysis. Authors hope that analysis outcome will be available soon and if the paper is accepted, they can be provided within the final version of the paper.

\section{Conclusions}

This paper reports the use of a remote laboratory facility to offer an undergraduate laboratory course within an engineering and engineering technology program. Offering a laboratory course over the Internet as a part of a regular program is a unique initiative. In addition to access to the experiments, the facility has an in-built evaluation and maintenance facility and a provision for monitoring client access profiles. In terms of hardware and software, the facility is composed of five independent modules: experiments, interfacing, GUI, sever, and client access. The modular approach allows the facility to be used for other courses/experiments without much change.

A number of measures were taken to ensure to identify any difficulties during the course offering process. For evaluation there are two approaches: one is to evaluate the system itself in terms of its user friendliness and effectiveness and the other is to evaluate students' learning outcomes. The first approach of the evaluation is incorporated within the facility as web applications, while the second approach involves formative and summative evaluation using a control group and a test group. A renounced researcher in teaching psychology and evaluation has also been hired as an external evaluator to ensure a credible outcome.

\section{Acknowledgement}

This paper is based on the project titled Design and Development of Internet-based Physical Laboratory Facility for an Undergraduate Course funded by the National Science Foundation under NSF Award Number DUE-0442374. Any opinions, findings and conclusions or recommendations expressed in this material are those of the authors and do not necessarily 
reflect the views of the National Science Foundation. The authors wish to thank Dr. Clifford Mirman, Chair of the Department of Technology, and Dr. Promod Vohra, Dean of the College of Engineering and Engineering Technology, for their administrative support.

\section{References}

1. A. P. Boyle, D. N. Bryon, \& C. R. C. Paul, Computer-based learning and assessment: A palaeontological case study with outcomes and implications, Computers and Geosciences, 23 (5), 573-580 (1997).

2. T. K. Grose, Can distance education be unlocked, PRISM, April, 19-23 (2003).

3. L. S. Chumbley, C. P. Hargrave, K. Constant, B. Hand, T. Andre, and E. A. Thompson, Project ExCEL: Web-based scanning electron microscopy for K -12 education, Journal of Engineering Education, April, 203-210 (2002).

4. A. K. Kamrani and S. M. Salhieh, Product design for modularity, Kluwer Academic Publishers, Boston, ISBN 0-7923-8554-3 (2000).

5. R. Safaric, D. W. Calkin, R. M. Parkin, and C. A. Czarnecki, Proceedings of the 6th UK Mechatronics Forum International Conference, Skovde, Sweden, September 9-11, 829-833 (1998).

6. C. C. Ko, B. M. Chen, S. Hu, V. Ramakrishnan, C. D. Cheng, Y. Zhuang, and J. Chen, A web-based virtual laboratory on a frequency modulation experiment, IEEE Transaction on Systems, Man, and Cybernetics, Part-C, Applications and Reviews, 31(3), 295-303 (2001).

7. M. Manasseh, E. Kausel, and K. Amaratunga, A web-accessible shaking table experiment for the remote monitoring of seismic effects in structures, Proceedings of the American Society of Engineering Education Annual Conference and Exposition, Salt Lake City, Utah (2004).

8. N. Chao and B. Mohr, Real hand-on laboratory experiments, anytime, anywhere, ASEE National Conference, Albuquerque, New Mexico, June 25, (2001).

9. H. A. Malki and A. Matarrita, Web-based control systems laboratory using LabVIEW, Journal of Engineering Technology, Spring, 22-25 (2003).

10. A. K. M. Azad and P. Nadakuditi (2006). Internet-based Facility for Physical Laboratory Experiments, Advanced Technology for Learning, 3(1), pp.29-35.

11. National Instruments, Measurement and Automation, http://www.ni.com/, (2004).

12. Special Issue: LabVIEW applications in engineering education, The International Journal of Engineering Education, 16(3), (2000).

13. J. Essick, Advanced LabVIEW Labs, Prentice-Hall, (1999).

14. J. Travis, Internet Applications in LabVIEW, Prentice-Hall, (2000).

15. A. K. M. Azad and X. Song, Internet-Based Laboratory Experiments as a Part of an Engineering Technology Program, Computers in Education Journal. (Scheduled to publish in January-March issue), (2007). 


\section{Appendix-A}

Instruction: Please prepare your post-laboratory report using the following instructions. The report must be within two pages of length.

Course title: Tech277A- Digital Logic Design Laboratory

Student name:

Laboratory number:

Dates of laboratory performance:

Date of post-laboratory submission:

Title: (Find a title, which should convey the substance of the task done under this laboratory session.)

Abstract: (It will be the brief summary of the work and results stated in concentrated form)

Conclusions: (It should include important points of the result [in details]. You also need to mention any problem experienced during the course of the laboratory session. Also discuss its possible cause and suggested remedy, whether it solved or not during the laboratory session.) 